\title{
Critical behavior of the hopping expansion from the Functional Renormalization Group
}

\section{R. Banerjee*}

Pittsburgh Particle Physics, Astrophysics, and Cosmology Center,

Department of Physics and Astronomy,

University of Pittsburgh, Pittsburgh, PA 15260, U.S.A

E-mail: rub18@pitt. edu

\begin{abstract}
A lattice version of the widely used Functional Renormalization Group (FRG) for the Legendre effective action is solved - in principle exactly - in terms of graph rules for the linked cluster expansion. Conversely, the FRG induces nonlinear flow equations governing suitable resummations of the graph expansion. The (finite) radius of convergence determining criticality can then be efficiently computed as the unstable manifold of a Gaussian or non-Gaussian fixed point of the FRG flow. The correspondence is tested on the critical line of the Lüscher-Weisz solution of the $\phi_{4}^{4}$ theory and its $\phi_{3}^{4}$ counterpart.
\end{abstract}

The 36th Annual International Symposium on Lattice Field Theory - LATTICE2018 22-28 July, 2018

Michigan State University, East Lansing, Michigan, USA.

\footnotetext{
* Speaker.
} 


\section{Introduction and Motivation}

The functional renormalization group (FRG) has become one of the most widely and fruitfully used techniques in quantum many body physics, and is now applied to areas as diverse as quantum gravity, particle physics, and solid state physics [1]. The FRG is a reformulation of quantum field theory that focusses on the non-linear response of functionals to a scale dependent mode modulation introduced by replacing the bare action $S[\chi]$ with $S[\chi]+\frac{1}{2} \chi \cdot R_{k} \cdot \chi$ in the functional integral. The regulator kernel $R_{k}$ supresses low energy modes and vanishes at $k=0$, such that the scale $k$ smoothly interpolates between the bare theory and the renormalized theory. Modern formulations focus on the Legendre effective action $\Gamma_{k}$, whose flow satisfies

$$
\partial_{k} \Gamma_{k}[\phi]=\frac{1}{2} \operatorname{Tr}\left\{\partial_{k} R_{k}\left[\Gamma_{k}^{(2)}+R_{k}\right]^{-1}\right\} .
$$

The versatility of the flow equation (1.1) is partly due to its kinematical nature; dynamical information is injected solely through initial conditions. As a consequence, fully non-perturbative results require some such initial conditions. An especially good choice are ultralocal initial conditions as they can, in a lattice formulation, be computed exactly from single site integrals [2]. A solution of (1.1) with such initial data, if feasible, will emulate a linked cluster or hopping expansion but with a scale dependent long-ranged interaction

$$
S[\chi]=\sum_{x} s\left[\chi_{x}\right]+\frac{\kappa}{2} \sum_{x, y} \chi_{y} \ell_{x y}(k) \chi_{x} .
$$

For definiteness we consider here a self-interacting, one-component, scalar field theory on a $D$ dimensional hypercubic lattice (identified with $\mathbb{Z}^{D}$ ) in a dimensionless formulation. Then, $s: \mathbb{R} \rightarrow$ $\mathbb{R}$ is a real even function bounded from below that collects all terms referring to a single site. The hopping parameter $\kappa>0$ is a dimensionless combination of the original mass, the coupling parameters, and the lattice spacing. A fundamental lattice action has the form (1.2) with a $k$ independent $\ell_{x y}$ that connects only nearest neighbors. In order to relate (1.1) to a hopping expansion we take $\kappa$ itself as the control parameter and replace (1.1) by

$$
\partial_{\kappa} \Gamma_{\kappa}=\frac{1}{2} \sum_{x, y} \ell_{x y}\left[\Gamma_{\kappa}^{(2)}+\kappa \ell\right]_{x y}^{-1}, \quad \Gamma_{\kappa}[\phi]=\Gamma_{0}[\phi]+\sum_{l \geq 2} \kappa^{l} \Gamma_{l}[\phi] .
$$

Here $\Gamma_{0}[\phi]=\sum_{x} \gamma\left(\phi_{x}\right)$, where $\gamma$ and its derivatives $\gamma_{n}$ are computable at a single site $x$ from $s$ only. The $O(\kappa)$ term vanishes, $\Gamma_{2}[\phi]=-\frac{1}{4} \sum_{x, y}\left(\ell_{x y}\right)^{2} \gamma_{2}\left(\phi_{x}\right)^{-1} \gamma_{2}\left(\phi_{y}\right)^{-1}$, and all $\Gamma_{l}, l \geq 3$, are then determined recursively. The direct recursion turns out to become intractable beyond $O\left(\kappa^{6}\right)$, say. However, a closed graph theoretical solution of the recursion can be obtained that yields $\Gamma_{l}$ for any $l \geq 1$ [3]. Importantly, the series in (1.3) can be expected to have finite radius of convergence $\kappa<\kappa_{c}$, at least as far as the associated vertex functions are concerned [9]. Once the series (1.3) has been constructed, an in principle exact solution of (1.1)'s lattice counterpart arises simply by substitution, $\Gamma_{k}=\left.\Gamma_{\kappa}\right|_{\ell \mapsto \ell(k)}$, for suitable $\ell(k)$ playing the role of $R_{k}$. This differs from the standard uses of the FRG [1] in the way initial conditions are imposed: $R_{k}$ is chosen such that $\Gamma_{k_{0}}[\phi]$, for some finite $k=k_{0}$, is (up to kinematical factors) determined by the above $\Gamma_{0}[\phi]=\sum_{x} \gamma\left(\phi_{x}\right)$. In overview, we propose to use the graph rule [3] for the computation of vertex (and other correlation) functions but determine bulk quantities from the FRGs (1.1), (1.3). 


\section{Critical behavior from the LPA's unstable manifold}

For the hopping expansion the locus of infinite correlation length (approached from the symmetric phase) is deemed to coincide with the radius of convergence $\kappa_{c}$ of the (two-point and then all other) susceptibilities. Traditionally, $\kappa_{c}$ has been estimated by pushing their hopping expansion to high orders, at considerable effort. Our proposed alternative rests on two simple observations: (i) any bulk quantity other than a susceptibility should give the same $\kappa_{c}$, in particular $\Gamma_{\kappa}$ for constant field (identified with the lattice average) should be a legitimate choice. (ii) For $\kappa<\kappa_{c}$, specialization to constant fields and resummation in (1.3) are commuting operations. The FRG (1.1) specialized to constant fields (up to kinematical factors) is known as the Local Potential Approximation (LPA), or its modified version LPA' (see first ref. in [1]). On general grounds, the relation between the bare and renormalized parameters can be found by injecting initial data (determined by the bare parameters) at the ultralocal scale $k=k_{0}$, and running the flow equation for (truncations of) $\Gamma_{k}$ to the fixed point at $k \approx 0$. This yields the correlated values of the bare parameters in the action tuned to ensure that $\Gamma[\phi]$ is based on the fixed point, i.e. the unstable manifold of the fixed point in question. In summary, one should be able to determine $\kappa_{c}$ from the unstable manifold of the LPA (or LPA') approximation to the FRG (1.1).

Explicitly, the following LPA ansatz $\Gamma_{k}\left[\phi_{0}\right]=a^{D} \sum_{x}\left\{-\frac{1}{2} \phi_{0}(x)\left(\Delta \phi_{0}\right)(x)+U_{k}\left(\phi_{0}(x)\right)\right\}$, is taken as the starting point, where $\Delta$ is the lattice Laplacian and $a$ the lattice spacing. The flow equation (1.1) then specializes to

$$
\partial_{k} U_{k}\left(\phi_{0}\right)=\frac{1}{2} \int_{-\pi / a}^{\pi / a} \frac{d^{D} p}{(2 \pi)^{D}} \frac{\partial_{k} R_{k}(p)}{\hat{p}^{2}+R_{k}(p)+U_{k}^{\prime \prime}\left(\phi_{0}\right)},
$$

where the $k$ differentiation is at fixed $\phi_{0}$ and $\hat{p}^{2}=\frac{4}{a^{2}} \sum_{j} \sin ^{2} \frac{p_{j} a}{2}$. Next we fix lattice units $(a=1)$ and choose $R_{k}(p)$ to be the lattice step function $R_{k}(p)=\left(k^{2}-\hat{p}^{2}\right) \theta\left(k^{2}-\hat{p}^{2}\right)$. Then

$$
k \partial_{k} U_{k}\left(\phi_{0}\right)=\frac{k^{2} \operatorname{Vol}(k)}{k^{2}+U_{k}^{\prime \prime}\left(\phi_{0}\right)}, \quad \operatorname{Vol}(k):=\int_{-\pi}^{\pi} \frac{d p}{(2 \pi)^{D}} \theta\left(k^{2}-\hat{p}^{2}\right) .
$$

The above flow equations and the fields have been dimensionful, and as such they do not lend themselves to a fixed point analysis. In particular the dimensionful LPA potential $U_{k}\left(\phi_{0}\right)$ will not itself reach a fixed point, but rather exhibit a characteristic scaling behavior. To proceed we transition to a dimensionless LPA formulation by rescaling both the field and potential,

$$
V_{k}(\phi):=\frac{1}{\mu_{D} k^{D}} U_{k}\left(\phi_{0}(\phi)\right), \quad \phi_{0}(\phi):=k^{\frac{D-2}{2}} \sqrt{\mu_{D}} \phi, \quad \mu_{D}:=\frac{1}{(4 \pi)^{D / 2} \Gamma\left(\frac{D}{2}+1\right)} .
$$

The new potential $V_{k}(\phi)$ is a dimensionless function of the dimensionless field $\phi$. The constants are adjusted such that only the dimensionless volume function $\operatorname{vol}(s):=\operatorname{Vol}\left(k_{0} s\right) /\left(\mu_{D} k_{0}^{D} s^{D}\right), 0 \leq$ $s:=k / k_{0} \leq 1, k_{0}:=\sqrt{4 D}$, occurs. It is determined numerically, and is roughly bell shaped with a maximum of $O(1)$. The normalizations are such that $\operatorname{vol}(0)=1$ and $\operatorname{vol}(1)=1 / \mu_{D} k_{0}^{D}$.

The dimensionful flow equation (2.2) translates into

$$
s \partial_{s} V_{s}(\phi)=-D V_{s}(\phi)+\frac{D-2}{2} \phi V_{s}^{\prime}(\phi)+\frac{\operatorname{vol}(s)}{1+V_{s}^{\prime \prime}(\phi)} .
$$


In this form one can now search meaningfully for a $s \rightarrow 0$ fixed point potential solving

$$
0=-D V_{*}(\phi)+\frac{D-2}{2} \phi V_{*}^{\prime}(\phi)+\frac{1}{1+V_{*}^{\prime \prime}(\phi)} .
$$

To proceed, insertion of the Taylor series $V_{s}(\phi)=\sum_{i \geq 0} \frac{g_{2 i}(s)}{(2 i) !} \phi^{2 i}$ into (2.4) produces the beta functions for the couplings $g_{2 i}(s)$,

$$
\begin{aligned}
s \partial_{s} g_{2 i} & =\beta_{2 i}\left(g_{2}, \ldots, g_{2 i+2}\right), \quad i \geq 1, \\
\beta_{2} & =-2 g_{2}-\frac{\operatorname{vol}(s)}{\left(1+g_{2}\right)^{2}} g_{4}, \quad \beta_{4}=(D-4) g_{4}-\frac{\operatorname{vol}(s)}{\left(1+g_{2}\right)^{2}} g_{6}+\frac{6 \operatorname{vol}(s)}{\left(1+g_{2}\right)^{3}} g_{4}^{2},
\end{aligned}
$$

etc. Truncating via $g_{2 N+2} \equiv 0$ at some order $N$ a closed system of $N+1$ ODEs arises. The lowest order equation $s \partial_{s} g_{0}=-D g_{0}+\operatorname{vol}(s) /\left(1+g_{2}\right)$ determines $g_{0}$ once $g_{2}$ is known.

Clearly, the behavior of the dimensionless couplings in the vicinity of the fixed point is instrumental for the critical behavior. The fixed couplings themselves obey a simple recursion relation of the form $g_{2 i}^{*}=-2 g_{2}^{*}\left(1+g_{2}^{*}\right)^{i} P_{i-2}\left(g_{2}^{*}\right)$, where generally $P_{n}$ is a polynomial of degree $n$. The value of $g_{2}^{*}$ is constrained by the truncation condition $g_{2 N+2}^{*}=0$, i.e. it is the root of the high order polynomial equation $2 g_{2}^{*}\left(1+g_{2}^{*}\right)^{n+1} P_{n-1}\left(g_{2}^{*}\right)=0$. In general this equation produces many spurious 'fixed points', but the 'correct' solution can be selected by requiring that its corresponding stability matrix,

$$
M\left(g^{*}\right)_{i j}:=\left.\frac{\partial \beta_{2 i}}{\partial g_{2 j}}\right|_{g=g^{*}},
$$

have precisely one negative eigenvalue, conventionally denoted by $-\theta_{1}$. For $D \geq 3$ one always has a Gaussian fixed point with $-\theta_{1}=-2$. In $D=3$ one finds in addition the Fisher-Wilson fixed point with $-\theta_{1} \approx-1.5396$.

The stability matrix $M$ determines the flow pattern in the vicinity of the fixed point. Writing $\delta g=\left(\delta g_{2}, \delta g_{4}, \ldots, \delta g_{2 N}\right)$ for a perturbation about the fixed point $g^{*}$ and expanding the truncated system (2.6) to linear order in the perturbation gives $s \partial_{s} \delta g=M\left(g^{*}\right) \delta g$. The solution of this linearized flow can be written in terms of the eigensystem $\left(v^{(j)},-\theta_{j}\right)$ of $M$ as

$$
\delta g(s)=\sum_{j=1}^{N} c_{j} v^{(j)} s^{-\theta_{j}},
$$

where the boundary constants $c_{1}, \ldots, c_{N}$ are set at some scale $0<s_{0} \ll 1$. For small $s$ and $c_{2} \neq 0$ the sum will be dominated by the $j=1$ term (as $-\theta_{1}<0$ ) and thus blow up, taking the couplings away from the fixed point. Conversely, the locus of linearized couplings that flow to $g^{*}$ for $s \rightarrow 0$ is characterized by $c_{2}=0$. More intrinsically, there exists a unique linear combination such that

$$
a_{0}+\sum_{i=1}^{N} a_{i} g_{2 i}(s)=\text { const } c_{2} s^{-\theta_{1}}, \quad 0<s \leq s_{0} \ll 1 .
$$

For $c_{2}=0$ this linear combination describes the "linearized unstable manifold", i.e. the codimension one hyperplane from which the couplings flow into the fixed point. The coefficients of the 
unstable manifold can be computed analytically for the Gaussian fixed point in $D=4$, and come out as $a_{0}=0, a_{i}=1 /\left[2^{i-1}(i-1) !\right]$, for $1 \leq i \leq N$.

So far no action-specific information has entered. The fixed point, the critical exponents, and the linearized unstable manifold are computable solely in terms of the field content (here: one scalar field), the dimensionality $D$, the nature of the truncation (here: LPA), and the mode modulator (here: the lattice step function). Action-specific information is in the present setting injected by specifying initial data $g_{2 i}(s=1)$, computed from the ultralocal part of the theory's action.

In order to obtain this intial data, we first note that at any scale $k$, the Legendre effective action $\Gamma_{k}$ in (1.1) satisfies the functional integro-differential equation

$$
e^{-\Gamma_{k}\left[\phi_{0}\right]}=\int \prod_{x} d \chi_{0}(x) e^{-S\left[\chi_{0}\right]-\frac{1}{2}\left(\phi_{0}-\chi_{0}\right) \cdot R_{k} \cdot\left(\phi_{0}-\chi_{0}\right)-\left(\phi_{0}-\chi_{0}\right) \cdot \frac{\delta \Gamma_{k}}{\delta \phi_{0}}}
$$

where $S\left[\chi_{0}\right]=\frac{1}{2} \sum_{x}\left\{-\chi_{0}(x)\left(\Delta \chi_{0}\right)(x)+m_{0}^{2} \chi_{0}(x)^{2}+\lambda_{0} \chi_{0}(x)^{4} / 12\right\}$ is the bare action, and '.' denotes a sum over lattice sites. Although this equation cannot in general be solved exactly, at the ultralocal scale $k_{0}=\sqrt{4 D}$ where $R_{k_{0}}(x, y)=k_{0}^{2} \delta_{x y}+\Delta_{x y}$, (2.10) transcribes to

$$
e^{-\sum_{x} U_{k_{0}}\left(\phi_{0}(x)\right)}=\int \prod_{x} d \chi_{0}(x) e^{-S_{0}\left[\chi_{0}\right]-\frac{k_{0}^{2}}{2}\left(\phi_{0}-\chi_{0}\right) \cdot\left(\phi_{0}-\chi_{0}\right)-\left(\phi_{0}-\chi_{0}\right) \cdot U_{k_{0}}^{\prime}} .
$$

Here $S_{0}\left[\chi_{0}\right]$ is the ultralocal part of the bare action, and $U_{k_{0}}$ is the effective potential at $k=k_{0}$. Since all the quantities in (2.11) are ultralocal, the functional integral factorizes. After transitioning to a dimensionless potential $V_{s}(\phi)$ viz. (2.3), and hopping parametrizing the bare action, we obtain the ordinary integro-differential equation satisfied by $V_{s=1}(\phi)$

$$
e^{-\operatorname{vol}(1)^{-1} V_{s=1}(\phi)}=\int d \chi e^{-\frac{1-2 \lambda-D \kappa}{4 D \operatorname{vol}(1) \kappa} \chi^{2}-\frac{\lambda}{(4 D \operatorname{vol}(1) \kappa)^{2}} \chi^{4}-\frac{1}{2 \operatorname{vol}(1)}(\phi-\chi)^{2}-\operatorname{vol}(1)^{-1}(\phi-\chi) V_{s=1}^{\prime}(\phi)} .
$$

Inserting the truncation ansatz $V_{s=1}(\phi)=\sum_{i=0}^{N} \frac{g_{2 i}(s=1)}{(2 i) !} \phi^{2 i}$ into (2.12) then determines the initial couplings $g_{2 i}(s=1)$ as functions of the bare parameters $\kappa, \lambda$ via exactly computable single site integrals.

With the initial data known, the integration of the flow equations (2.6) (truncated at some order $N)$ proceeds as follows. Since the initial data $g_{2 i}(s=1), 1 \leq i \leq N$, are prescribed functions of $\kappa, \lambda$, a well-defined evolution via the ODE system will render the $g_{2 i}(s)=g_{2 i}(s \mid \kappa, \lambda)$ parametrically dependent on $\kappa, \lambda$ for all $s$ for which the evolution is regular. For generic $\kappa, \lambda$ the flow will not come close to the fixed point; it will do so however once it reaches the linearized unstable manifold at some $0<s_{0} \ll 1$. In a given polynomial approximation of order $N$ one therefore needs to solve

$$
a_{0}+\sum_{i=1}^{N} a_{i} g_{2 i}\left(s_{0} \mid \kappa, \lambda\right)=0 \quad \Longrightarrow \quad \kappa=\kappa_{c}(\lambda) .
$$

In the present context this will ensure that the flow very nearly reaches the fixed point, with limitations only set by numerical accuracy.

\section{Results for $\phi_{3}^{4}$ and $\phi_{4}^{4}$}

The above technique has been applied to determine $\kappa_{c}(\lambda)$ for $\phi^{4}$ theories in both $D=3$ and $D=4$. The shooting technique has been implemented in Mathematica without encountering 
significant obstructions from stiffness for reasonably large $N$. Related results have been obtained in [4], [5] in a different LPA formulation but without relation to (1.3) and the hopping expansion's radius of convergence.

As a proof of principle we first applied the shooting technique in $D=3$, aiming at the FisherWilson fixed point. Since in $D=3$ the anomalous dimension $\eta$ is non-zero, the neglection of a wavefunction renormalization constant in the LPA (as opposed to the LPA' ansatz) induces a systematic error. Nevertheless, the comparison of the LPA results with Monte-Carlo data [7] shows reasonable agreement.

\begin{tabular}{|c|c|c||c|c|c|}
\hline$\lambda$ & $\kappa_{c, M C}$ & $\kappa_{c}$ & $\lambda$ & $\kappa_{c, M C}$ & $\kappa_{c}$ \\
\hline 0.1 & 0.37341 & 0.3732 & 0.9 & 0.38451 & 0.3854 \\
0.2 & 0.3884 & 0.3882 & 1.3 & 0.36522 & 0.3659 \\
0.4 & 0.3975 & 0.3975 & 1.4 & 0.36028 & 0.362 \\
0.7 & 0.39253 & 0.3926 & 1.5 & 0.3553 & 0.358 \\
0.8 & 0.3887 & 0.3898 & 2.5 & 0.3134 & 0.3149 \\
\hline
\end{tabular}

Table 1: Critical values for $\phi_{3}^{4}$ theory in $D=3$. Left, $\kappa_{c, M C}$ from [7] with only significant digits displayed. Right $\kappa_{c}$ from LPA at truncation order $N=20$. The LPA errors are a combination of numerical and estimated truncation effects, only significant digits are displayed. The discrepancy can plausibly be attributed to the neglected anomalous dimension.

In $D=4$ only the Gaussian fixed point is found. By working with the LPA the $\eta=0$ contention [8] is probed for self consistency. As an illustration of the shooting technique we depict in Figs. 1(a) and 1(b) the flow of the couplings $g_{2}(s), g_{4}(s), g_{6}(s), g_{8}(s)$, and $g_{10}(s)$ in the $\phi_{4}^{4}$ theory towards the Gaussian fixed point. The truncation order is $N=20$, with $\kappa$ adjusted at fixed $\lambda$ such that the $g_{2 i}\left(s_{0}=0.001 \mid \kappa, \lambda\right)$ satisfy (2.13), with coefficients $a_{0}=0, a_{i}=1 /\left[2^{i-1}(i-1) !\right]$, for $1 \leq i \leq N$.

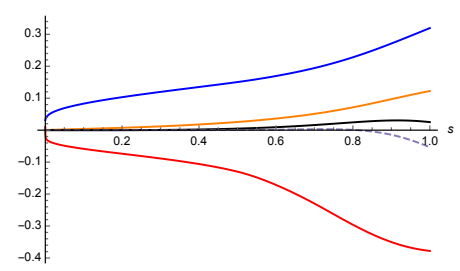

(a)

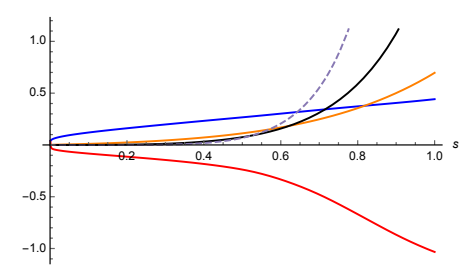

(b)

Figure 1: $\phi_{4}^{4}$ : flow of couplings $g_{2}(s), g_{4}(s), g_{6}(s), g_{8}(s), g_{10}(s)$ for $($ a) $(\lambda, \kappa)=(0.48548,0.2828)$, and $(b)(\lambda, \kappa)=(4.3303,0.1834)$. Red: $g_{2}$, Blue: $g_{4}$, Orange: $g_{6}$, Black: $g_{8}$, Dashed: $g_{10}$.

As noted earlier, the critical line $\kappa_{c}(\lambda)$ has been previously computed from the radius of convergence of the hopping expansion in [6]. A comparison of our results with the $\kappa_{c}(\lambda)$ values of Lüscher-Weisz (taken from Table 1 in [6]) is presented in Table 2.

In summary, the critical behavior in the hopping expansion, traditionally set by the radius of convergence [6], [9], can alternatively be obtained simply from the LPA or LPA' approximation to the FRG. 


\begin{tabular}{|c|c|c|}
\hline$\lambda$ & $\kappa_{c, L W}$ & $\kappa_{c} / 2$ \\
\hline 0 & $0.1250(1)$ & $0.1250(1)$ \\
$2.4841 \times 10^{-2}$ & $0.1294(1)$ & $0.12928(3)$ \\
$3.5562 \times 10^{-2}$ & $0.1308(1)$ & $0.13068(3)$ \\
$1.3418 \times 10^{-1}$ & $0.1385(1)$ & $0.1381(4)$ \\
$2.7538 \times 10^{-1}$ & $0.1421(1)$ & $0.1416(4)$ \\
$4.8548 \times 10^{-1}$ & $0.1418(1)$ & $0.1414(4)$ \\
$7.7841 \times 10^{-1}$ & $0.1376(1)$ & $0.1374(4)$ \\
1.7320 & $0.1194(1)$ & $0.1190(5)$ \\
2.5836 & $0.1067(1)$ & $0.1066(5)$ \\
4.3303 & $0.09220(9)$ & $0.0917(7)$ \\
$\infty(\mathrm{LW})$ or $100(\mathrm{LPA})$ & $0.07475(7)$ & $0.0722(1)$ \\
\hline
\end{tabular}

Table 2: Critical values for $\phi_{4}^{4}$ theory in $D=4$. Left, $\kappa_{c, L W}$ from Lüscher-Weisz [6]. Right $\kappa_{c} / 2$ from LPA at truncation order $N=20$. The errors in the LPA results are a combination of numerical and estimated truncation errors.

Acknowledgements: Support by a PITTPACC fellowship, and University of Pittsburgh A\&S$P B C, A \& S-G S O$ travel grants is gratefully acknowledged. With many thanks to Max Niedermaier.

\section{References}

[1] P. Kopietz, L. Bartosch, and F. Schütz, Introduction to the Functional Renormalization Group, Springer, 2010.

A. Wipf, Statistical Approach to Quantum Field Theory, Springer 2013.

R. Percacci, An introduction to covariant Quantum Gravity and Asymptotic Safety, World Scientific, 2017.

[2] T. Machado and N. Dupuis, From local to critical fluctuations in lattice models: a nonperturbative renormalization group approach, Phys. Rev. E82, 041128 (2010).

[3] R. Banerjee and M. Niedermaier, Graph rules for the linked cluster expansion of the Legendre effective action, under review, March 2018.

[4] J-M. Caillol, Critical line of the $\Phi^{4}$ theory on a simple cubic lattice in the local potential approximation, Nucl. Phys. B865 , 2 (2012).

[5] J-M. Caillol, Critical line of the $\Phi^{4}$ theory on a $4 D$ cubic lattice in the local potential approximation, Condensed Matter Physics, (2013), Vol. 16, No. 4, 4300.

[6] M. Lüscher and P. Weisz, Scaling laws and triviality bounds in the lattice $\phi^{4}$ theory, Nucl. Phys. B290, 25 (1987).

[7] M. Hasenbusch, A Monte Carlo study of leading order scaling corrections of $\phi^{4}$ theory on a three dimensional lattice, J. Phys. A: Math. Gen. 32 (1999) 4851.

[8] O. Rosten, Triviality from the exact renormalization group, JHEP 07 (2009) 019.

[9] A. Pordt and T. Reisz, Linked cluster expansions beyond nearest neighbor interactions: convergence and graph classes, Int. J. Mod. Phys. A12 (1997) 3739. 